Regular Paper

\title{
Magnetic Field and Oscillating Analysis of Hybrid Suspension Systems Combining Magnetic Spring and Damper
}

\author{
Kenshiro UTSUNOMIYA ${ }^{* 1}$, Takashi TODAKA ${ }^{* 1}$ and Masato ENOKIZONO ${ }^{* 1}$
}

\begin{abstract}
This paper presents results obtained in development of a hybrid suspension system combining functions of a magnetic spring and a magnetic damper. In the parallel connection of the developed magnetic suspension system with a metal spring, it is possible to make a soft spring near the middle point supporting a large mass by combining the negative spring constant with the positive one. The dynamic vibration characteristics of the developed hybrid suspension system are demonstrated.
\end{abstract}

Keywords: magnetic spring, magnetic damper, suspension system, permanent magnet.

(Received: 31 May 2012, Revised: 14 June 2013)

\section{Introduction}

Vibration control devices utilizing various kinds of metal springs, air suspensions, and oil dampers have been developed as a measure to settle vibration problems arising from structures, machines and equipments. The role of dampers is to absorb impact forces by generating a damping force, which is proportional to velocity in the opposite direction of the movement and displacement. Usually the damping force can be easily obtained by the Coulomb friction force occurred from viscosity of oil, however there are disadvantages and environmental limits to use oil such as oil leakage. On the other hand, the magnetic dampers have advantages such as clean, maintenance free, and no environmental limitation because the damping force can be generated by the Lorentz force due to eddy currents.

In addition, magnetic springs utilizing permanent magnets have a negative spring constant because the force is inversely proportional to displacement against metallic springs, which have a positive spring constant governed by the Hooke's law. Therefore it is possible to

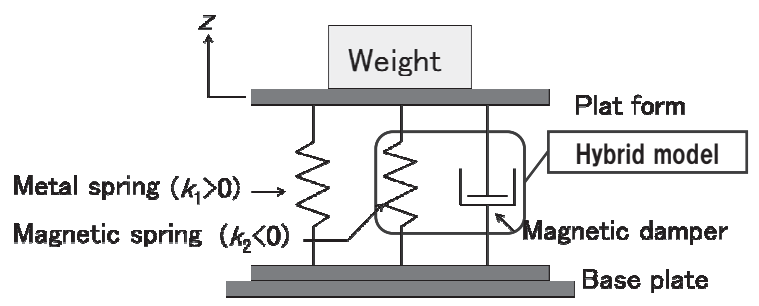

Fig. 1. Hybrid suspension system.

\footnotetext{
Correspondence: K.UTSUNOMIYA, Department of Electrical and Electric Engineering, Faculty of Engineering, Oita University, 700 Dannoharu, Oita 870-1192, Japan email: v12e2006@oita-u.ac.jp

${ }^{* 1}$ Oita University
}

make a soft spring system at the middle point around supporting a loaded mass by connecting a metallic spring and a magnetic spring in parallel as shown in Fig. 1. As a result, a lower resonant frequency can be realized. In this paper, we propose a new hybrid magnetic suspension system combined a magnetic spring with a magnetic damper, and the basic feature and the dynamic vibration characteristic were analyzed by using the finite element method [1]-[4].

\section{Finite Element Analysis and Moving Simulation}

The hybrid model consists of an axisymmetric cylindrical magnetic spring and an eddy current damper. The electromagnetic field distributions of the hybrid model were analyzed by using the three-dimensional axisymmetric finite element method. The eddy current for producing the damping force is given by Eq. (1) and the governing equation of the axisymmetric magnetic field expressed by the magnetic vector potential is written as Eq. (2):

$$
\boldsymbol{J}_{\mathrm{e}}=\sigma(\boldsymbol{V} \times \boldsymbol{B}),
$$

$$
\frac{\partial}{\partial r}\left\{\frac{v_{z}}{r} \frac{\partial}{\partial r}\left(r A_{\theta}\right)\right\}+\frac{\partial}{\partial z}\left(v_{r} \frac{\partial A_{\theta}}{\partial z}\right)=-v_{0} r o t \boldsymbol{M}-\sigma(\boldsymbol{V} \times \boldsymbol{B}),
$$

where $\boldsymbol{J}_{\mathrm{e}}$ is the eddy current flowing in a conductive mover, $\boldsymbol{v}$ the velocity of the mover, $\boldsymbol{B}$ the magnetic flux density, $\sigma$ the conductivity, $A_{\theta}$ the magnetic vector potential, $\boldsymbol{M}$ the magnetization of magnets, $v_{\mathrm{i}}(i=r, z)$ the magnetic resistivity, and $v_{0}$ the magnetic resistivity in vacuum.

The conducting mover and the magnetic spring have the same displacement and they move simultaneously. The electromagnetic force is generated for the mover by the magnetic field of the stationary magnets and the eddy current. At the same time the electromagnetic force acting on the magnetic spring is generated accordance with the position of the moving magnets and the stationary magnets. 
In order to calculate the electromagnetic force acting on the conductive mover of the eddy current damper, the Maxwell's tensor method is used after obtaining the magnetic field distributions calculated with the time domain finite element method. In addition, free vibration and forced vibration simulations to clarify the dynamic performance of the hybrid suspension system were performed with the step-by-step method by using the discretized motion equation in time domain. In the simulation, the moving direction was assumed to be in $\mathrm{z}$-direction and the Coulomb frictions at supports were neglected for convenience. Table 1shows the conditions used in the analysis.

\section{Hybrid Suspension Systems Combining Magnetic Spring and Damper}

\subsection{Fundamental Hybrid Model}

Fig. 2 shows the hybrid model of the magnetic spring and the magnetic damper utilizing permanent magnets. The magnetic spring, which has a negative spring constant in vicinity of the middle point, consists of the moving magnets and the stationary magnets. The magnetic damper consists of the stationary magnets, the cylindrical copper mover and the back yoke. The damping force is generated due to eddy currents flowing in the copper mover and the magnetic field generated by the stationary permanent magnets. [5]

Fig. 3 shows the magnetic flux distribution of the hybrid model at $z=0 \mathrm{~mm}$ (a) and $z=5 \mathrm{~mm}$ (b) when $v$ $=0(\mathrm{~m} / \mathrm{sec})$. In this axisymmetric analysis, the Dirichlet boundary condition $\left(A_{\theta}=0\right)$ was assumed on the $z$ axis. On the other boundaries, the Neumann boundary condition was assumed. In comparing Fig. 3 (a) with Fig. (b),

Table 1 Conditions used in the analysis

\begin{tabular}{|c|c|}
\hline $\begin{array}{l}\text { Residual magnetization of } \\
\text { permanent magnet }[\mathrm{T}]\end{array}$ & 1.26 \\
\hline Yoke material & SS400 \\
\hline Conductivity of the yoke $[\mathrm{S} / \mathrm{m}]$ & $1.02 \times 10^{7}$ \\
\hline Conductivity of the mover $[\mathrm{S} / \mathrm{m}]$ & $5.85 \times 10^{7}$ \\
\hline Displacement $[\mathrm{mm}]$ & $\begin{array}{l}\text { from }-15 \text { to } 15 \\
\text { (1mm step) }\end{array}$ \\
\hline Velocity of the mover $[\mathrm{m} / \mathrm{sec}]$ & $\begin{array}{c}\text { from }-1 \text { to } 1 \\
(0.1 \mathrm{~m} / \mathrm{sec} \text { step })\end{array}$ \\
\hline
\end{tabular}

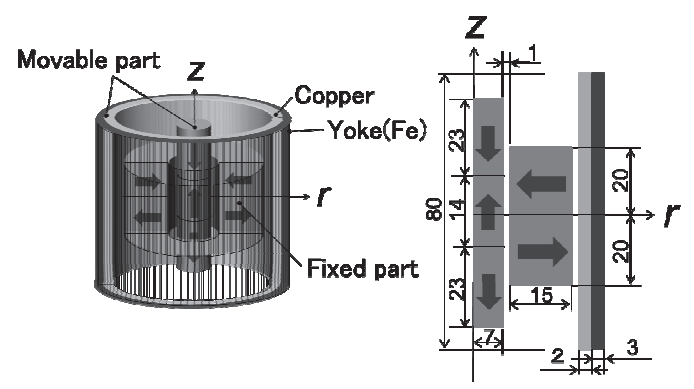

Fig. 2. Construction of the hybrid model. the force acting on the moving magnets has the same direction of the displacement, because the attractive force works in parallel to the flux lines.

Fig. 4 shows the characteristic of the elastic forces. As shown in this figure, the metal spring has a positive spring constant and it is supporting a loaded mass at the middle point $(z=0)$. As the force generating in the magnetic spring is proportional to the displacement near $z=0$, the spring constant is opposite to that of the metal spring and it means negative value. By combining the metal spring with the magnetic one in parallel, a soft spring that has a very small spring constant can be configured in vicinity of the middle point at $z=0 \mathrm{~mm}$.
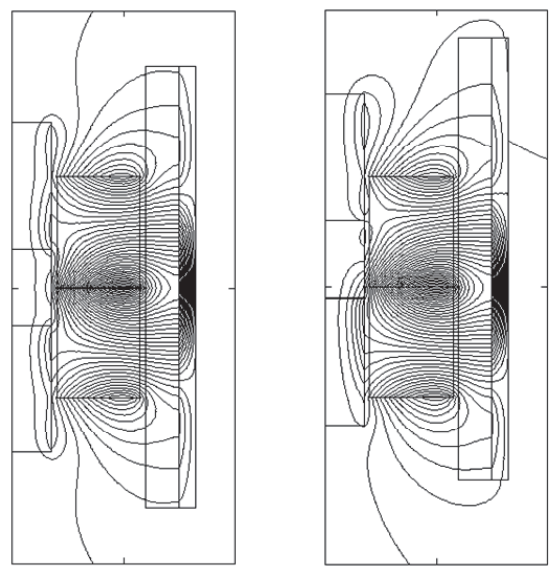

Fig. 3. Magnetic flux distributions.

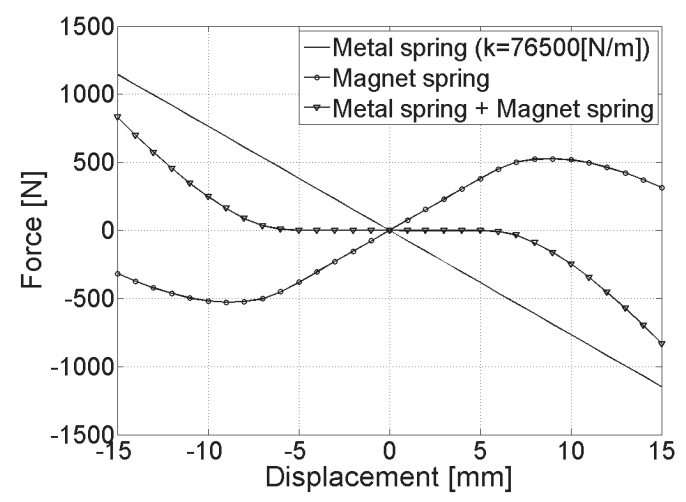

Fig. 4. Elastic force characteristics.

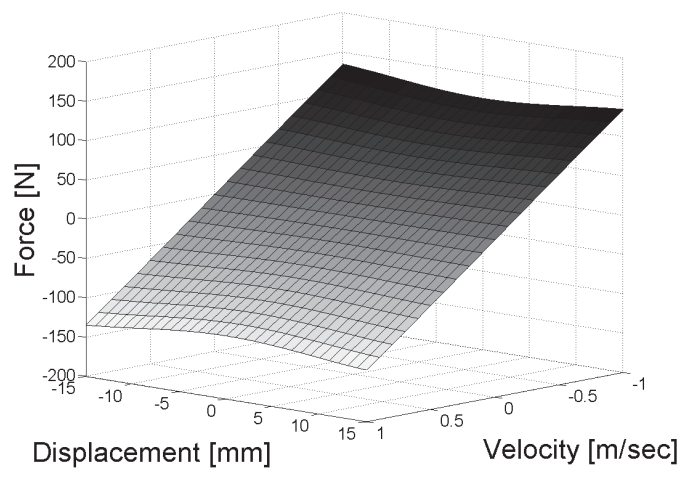

Fig. 5. Damping force characteristic. 
Fig. 5 shows the characteristics of the damping force acting on the copper cylindrical mover and the back yoke. The damping force becomes a function of displacement (position) and velocity, because the magnetic flux density distribution is not uniform. The damping force at $z=0 \mathrm{~mm}$ was $12.2 \mathrm{~N}$ when $v=-0.1 \mathrm{~m} / \mathrm{sec}$.

Fig. 6 shows a result of a free vibration simulation based on the elastic force and damping force characteristics mentioned in the above (Figs. 4 and 5). In this simulation we assumed that thickness of the copper cylinder was $2 \mathrm{~mm}$, one of back yoke was $3 \mathrm{~mm}$, and the initial velocity of the base plate at $\mathrm{t}=0$ was 0.1 $\mathrm{m} / \mathrm{sec}$. As shown in Fig. 6, we can point out that it is necessary to increase the damping force in order to attenuate oscillation in a short time by optimizing the copper cylinder and the back yoke.

Fig. 7 illustrates the vibration transmissibility characteristic of the suspension system obtained through vibration simulations forced by sinusoidal oscillations. The resonant frequency was $0.7 \mathrm{~Hz}$ and the maximum transmissibility was 1.36 . In order to reduce the vibration transmissibility more at resonance and improve the damping force characteristic, we tried to optimize thickness of the copper cylinder firstly. The results will be discussed in the next section.

\subsection{Optimization of Thickness of the Copper Cylinder}

The damping force of the hybrid model is proportional to eddy currents flowing in the copper cylinder. It is evident that the interlinking flux in the cross section

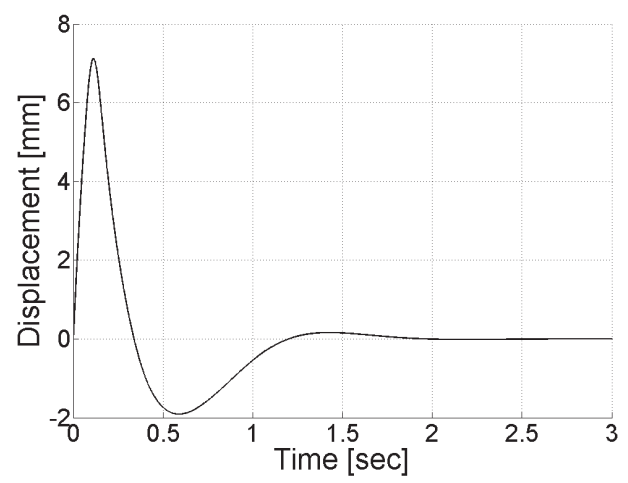

Fig. 6. Free oscillating simulation of the hybrid model.

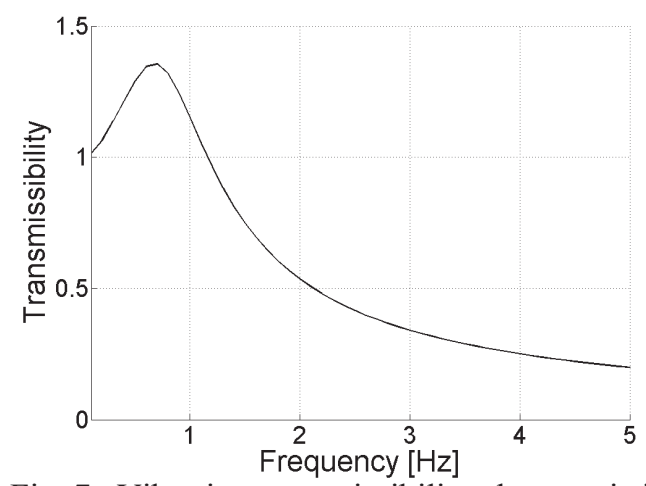

Fig. 7. Vibration transmissibility characteristic. of the copper cylinder can be increased by shortening distance between the stationary permanent magnets and the back yoke. However, the thickness of the copper cylinder becomes narrower and the volume flowing eddy currents decreases. Inversely, as the thickness of the copper cylinder becomes thicker, the volume flowing eddy currents increases but the interlinking flux and the magnetic flux density in the cross-sectional area of the cylinder decrease, because the distance between the stationary permanent magnets and the back yoke increases.

To find out an optimal condition, the thickness of the copper cylinder, $W$ was changed from $1 \mathrm{~mm}$ to 10 mm. Fig.8 shows the model used in the analysis. During this investigation, the thickness of the back yoke was kept to be $3 \mathrm{~mm}$, and the air gap between the stationary magnets and the inner surface of the copper cylinder was assumed to be $1 \mathrm{~mm}$.

Fig. 9 illustrates the damping force as a function of the thickness of the copper cylinder at $\mathrm{z}=0 \mathrm{~mm}$ and $\mathrm{v}=$ $-0.1 \mathrm{~m} / \mathrm{sec}$. As shown in this figure the damping force became the maximum when $W=6 \mathrm{~mm}$.

Figs.10 (a), (b) and (c) show the magnetic flux density distributions at $W=2,6$, and $10 \mathrm{~mm}$, respectively. In the case of $W=2 \mathrm{~mm}$ (a), the magnetic flux density in the copper cylinder was high because the distance between the stationary permanent magnets and the back yoke was narrow. However, the damping force was small because of the narrow cross-sectional area of the copper cylinder. In the case of $W=10 \mathrm{~mm}$ (c), the magnetic flux density in the copper cylinder became lower and the damping force got smaller in comparison with those of $W=6 \mathrm{~mm}$ (b), as prospected.

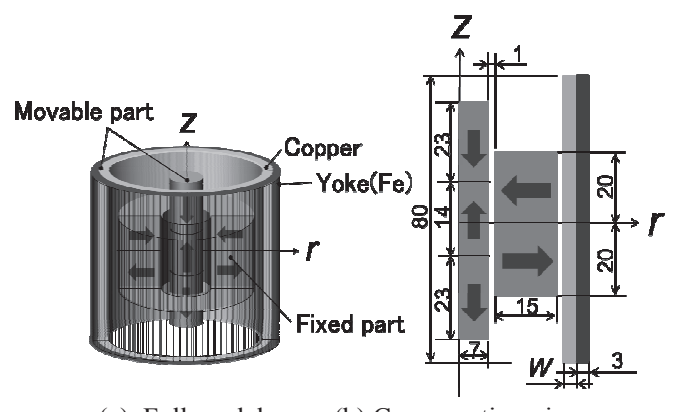

$\begin{array}{ll}\text { (a) Full model } & \text { (b) Cross-section view }\end{array}$

Fig. 8. Analysed hybrid model.

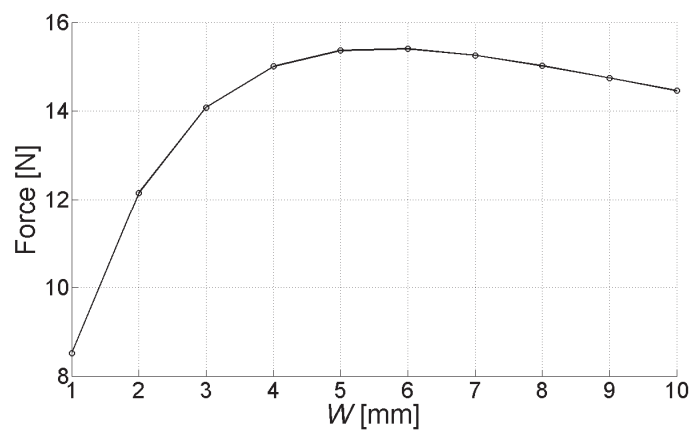

Fig. 9. Damping force characteristic depending on the thickness of the copper cylinder. 


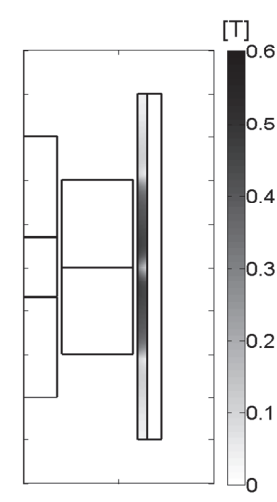

(a) $W=2 \mathrm{~mm}$

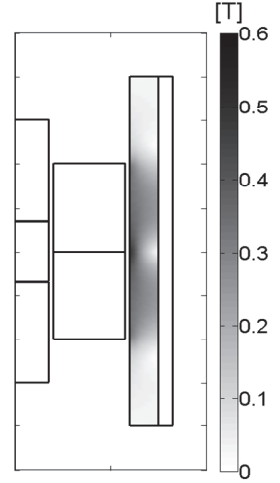

(b) $W=6 \mathrm{~mm}$

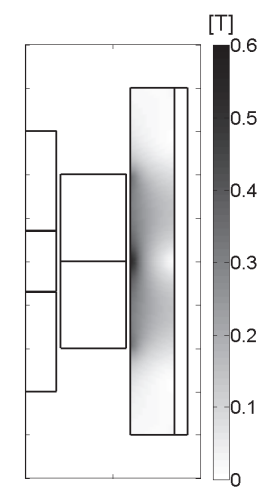

(c) $W=10 \mathrm{~mm}$

Fig. 10. Distributions of the magnetic flux density.

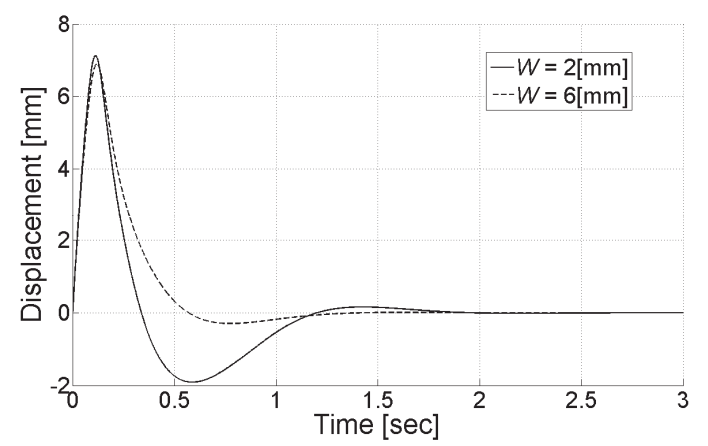

Fig. 11. Free oscillating simulation of the $2 \mathrm{~mm}$ and $6 \mathrm{~mm}$ thick copper cylinder models.

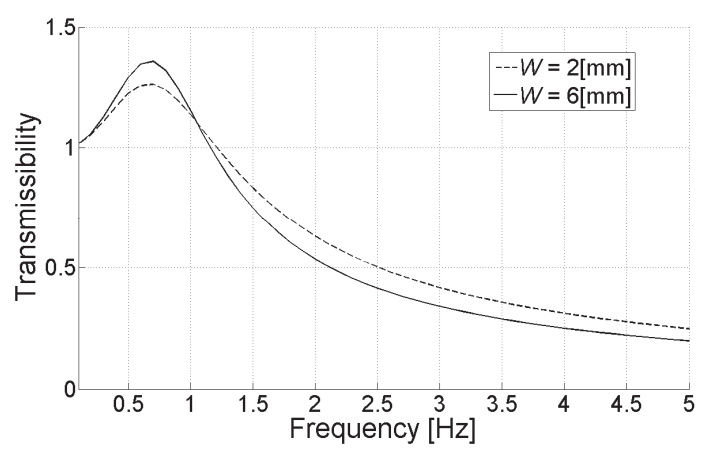

Fig. 12. Comparison of transmission characteristics.
Fig. 11 shows free vibration attenuation characteristics of the $2 \mathrm{~mm}$ and $6 \mathrm{~mm}$ thick copper cylinder models. In the analysis, the spring constant of the metal spring combined with the hybrid model in parallel was assumed to be $74800 \mathrm{Nsec} / \mathrm{m}$. As shown in this figure, attenuation characteristic is improved with the optimized model ( $W=6 \mathrm{~mm}$ ). Fig. 12 shows comparison of transmission characteristics obtained from the forced vibration simulations for each model. The resonant frequency of the optimized $6 \mathrm{~mm}$ thick copper cylinder model was $0.7 \mathrm{~Hz}$ and the vibration transmissibility at the resonance was 1.26. We call this model "model-A" hereafter.

As mentioned in the above, we could successfully improve the damping characteristic of the hybrid model by optimizing the copper cylinder thickness. Because the other parameters were neglected in the investigation, we should considerer more effective constructions to improve damping force and settle oscillation in the free vibration attenuation shown in Fig. 11.

\section{Improvement of the Stationary Magnet Part}

\subsection{Halbach Array Model}

In order to further improve the damping force, we applied the Halbach array to the stationary permanent magnet part as shown in Fig. 13. The Halbach array produces large magnetic flux density, therefore significant increase of the damping force can be expected. In addition, the elastic force characteristics associated with the application of the Halbach array are investigated to clarify the basic anti-vibration characteristics. Here we assumed that the height of the middle magnet in the Halbach array was equal to $H$ as shown in Fig. 13 and $H$ was changed from $2 \mathrm{~mm}$ to $20 \mathrm{~mm}$ per $2 \mathrm{~mm}$ step. During this investigation, the total amount of the stationery permanent magnets was kept to be constant.

\subsection{Damping Force Characteristics}

Fig. 14 shows the damping force characteristic of the hybrid model utilizing the Halbach array depending on the height of the middle magnet $H$ at $\mathrm{z}=0 \mathrm{~mm}$ and $\mathrm{v}=$ $0.1 \mathrm{~m} / \mathrm{sec}$. As shown in this figure, the damping force increased with increasing the height $H$ up to $18 \mathrm{~mm}$, however it was saturated over $18 \mathrm{~mm}$. Also the damping force was doubled comparing with the previous model (Fig. 9).

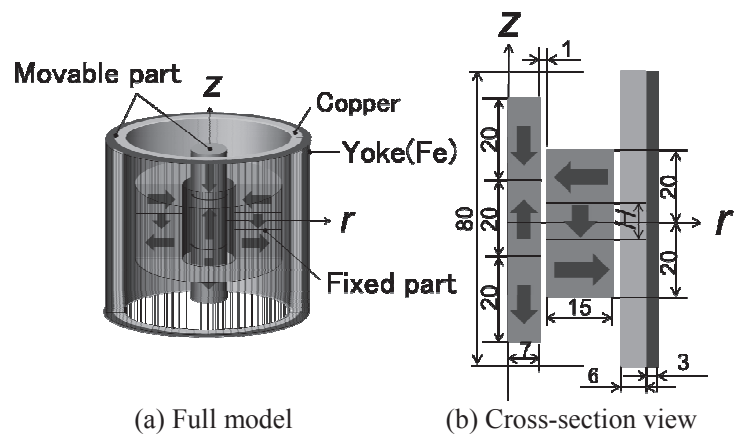

Fig. 13. Halbach array model. 
Fig. 15 shows the magnetic flux density distributions in the cross-sectional area of the copper cylinder in the case of $H=2,8,14$, and $20 \mathrm{~mm}$ when $z=0 \mathrm{~mm}$ and $v=0 \mathrm{~m} / \mathrm{sec}$. It is evident that the magnetic flux density increased with increasing the height $H$. However, when $H$ over $14 \mathrm{~mm}$, the increment of the magnetic flux density became smaller and the flux tended to concentrate in narrow regions.

\subsection{Elastic Force Characteristics}

Fig. 16 shows the relationship between the displacement of the magnetic spring and the magnetic force acting on the inner moving magnet. As shown in this figure, the strokes of the negative spring constants were shortened as the height $H$ got larger.

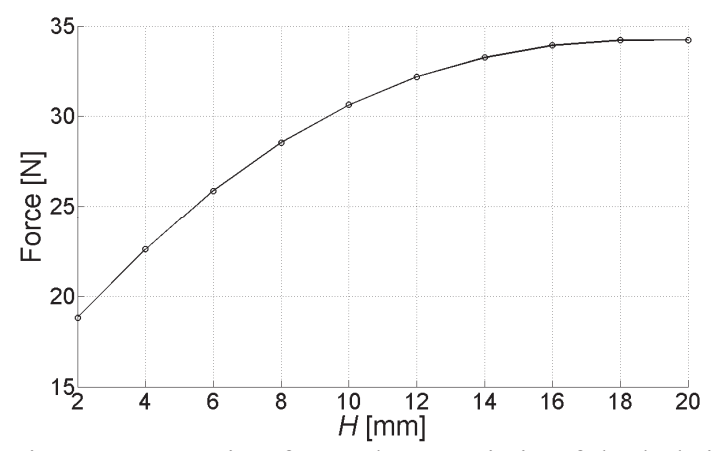

Fig. 14. Damping force characteristic of the hybrid model utilizing the Halbach array.

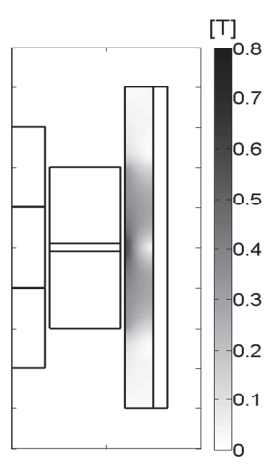

(a) $H=2 \mathrm{~mm}$

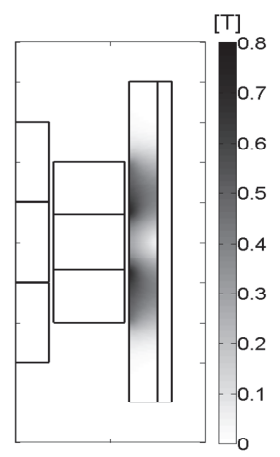

(c) $H=14 \mathrm{~mm}$

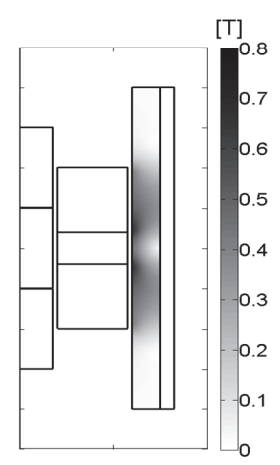

(b) $H=8 \mathrm{~mm}$

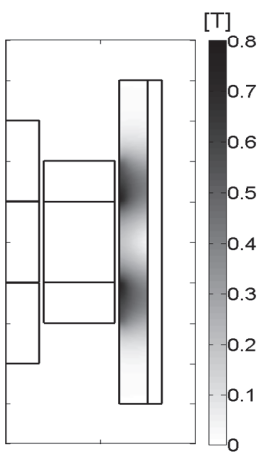

(d) $H=20 \mathrm{~mm}$
Fig. 15. Distributions of the magnetic flux density.
Fig. 17 shows the elastic force characteristics when the hybrid model (Fig. 13) was connected with the metal spring. The results was also compared with that of the model-A (Fig. 8: $W=6 \mathrm{~mm}$ ) near $z=0 \mathrm{~mm}$. As shown in this figure, the $4 \mathrm{~mm}$ height model had a quite long stroke keeping a soft spring constant and it is similar to the previously optimized hybrid model (model-A). In the case of $H=4 \mathrm{~mm}$, the spring constant was almost zero and the nonlinearity was weak in the range from -4 $\mathrm{mm}$ to $+4 \mathrm{~mm}$. We call this model "model-B" hereafter.

\subsection{Vibration Isolation Characteristics}

To make clear vibration isolation characteristics, free oscillating simulations based on the vibration damping characteristics and the elastic force characteristics discussed previously, were carried out by using the step-by-step method.

Fig. 18 shows comparisons of attenuation characteristics of the model-A and the model-B. The displacement of the model-B showed over-attenuation because the damping force was quite large in comparison with that of the model-A, which showed the damped oscillation. In this simulation, we assumed the initial velocity $\left(\mathrm{v}_{0}=0.1 \mathrm{~m} / \mathrm{sec}\right)$ of the base plate at $\mathrm{t}=0$ as a disturbance, thus the vibration time was longer due to large damping force. However in practice, the mover doesn't move so much for a sinusoidal one $(0.7 \mathrm{~Hz})$ because of large damping force.

Fig. 20 shows the characteristics of the vibration transmissibility obtained from the forced vibration simulations. The resonant frequency was $0.2 \mathrm{~Hz}$ and the vibration transmissibility at resonance was 1.04 .

From the results discussed in the above, it was clarified that applying the Halbach array to the stationary portion was effective to improve damping forces, to configure a more soft spring, to widen the stroke and to weaken the nonlinearity. It was also possible to shift the resonant frequency to lower side.

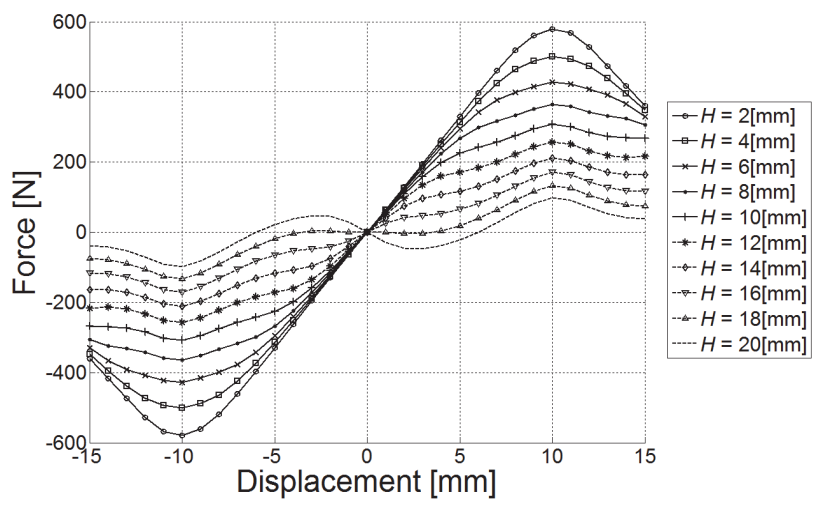

Fig. 16. Relationship between the displacement of the magnetic spring and the magnetic force acting on the inner moving magnet depending on the height of the mid magnet in Halbach array. 


\section{Conclusion}

In this paper, the hybrid suspension models combining the functions of the magnetic spring and the magnetic damper were proposed and the fundamental vibration characteristics were revealed. Firstly we optimized the thickness of the copper cylinder in the eddy current damper part. The suitable thickness was 6 $\mathrm{mm}$ and we could successfully improve the damping characteristic of the hybrid model. However the result of the free-oscillating simulation showed the quite long damped oscillation.

In the second step, to improve the attenuation characteristic, we applied the Halbach array to the stationary permanent magnet part. As a result, we could improve the damping force significantly by increasing the magnetic flux density. It was also possible to configure a softer spring having week nonlinearity in vicinity of the middle point. Adjustment of damping force, which is suitable for various applications and development of more compact structure models for practical applications of the developed hybrid suspension system remain as a further problem.

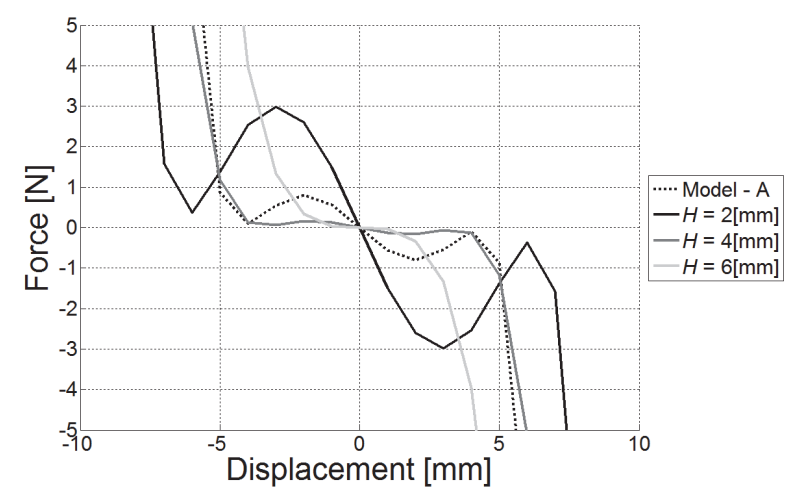

Fig. 17. Comparison of elastic force characteristics.

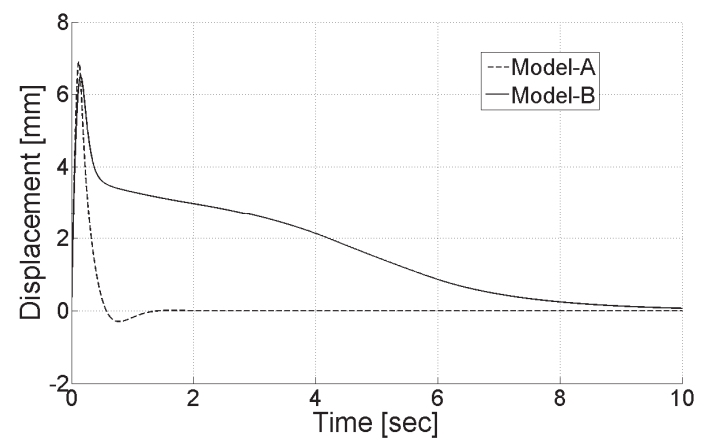

Fig. 18. Comparison of free oscillating simulation of the model-A with one of the model-B (the initial velocity $\mathrm{v}_{0}$ $=0.1 \mathrm{~m} / \mathrm{sec})$.

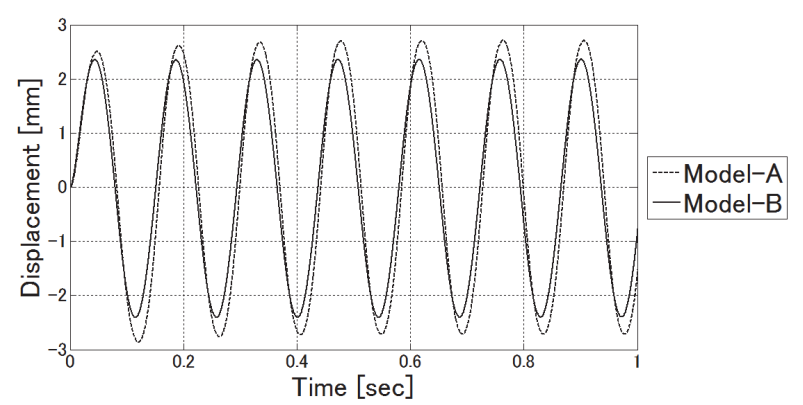

Fig. 19. Comparison of forced oscillating simulation of the model-A with one of the model-B ( frequency of $0.7 \mathrm{~Hz}$ ).

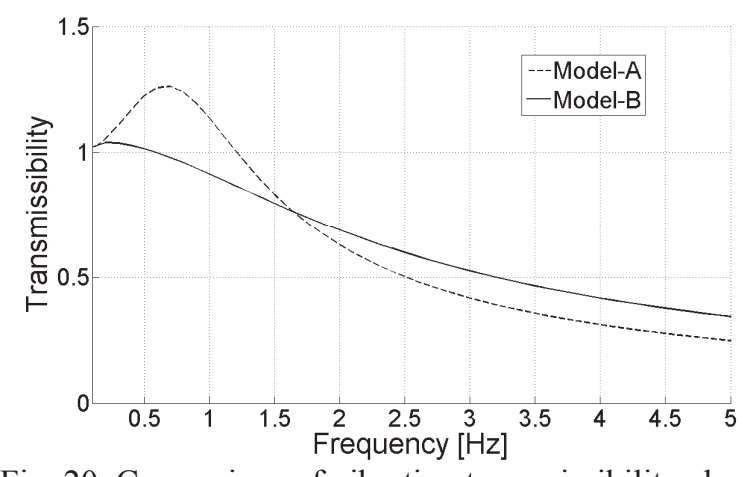

Fig. 20. Comparison of vibration transmissibility characteristic of the model-A with one of the model-B.

\section{References}

[1] K. Kuwata, S. Kojima, H. Ohima, E. Sugimoto, Y. Ogura, E. Fujita, M. Enokizono and S. Kaneko, "Development of Vibration Control Device and Shaker using Magnet spring, " J. JSAEM, Vol. 17, pp. 557-564, 2009.

[2] H. Ohshimo, E. Fujita, Y. Enoki and S. Wagata, "An Experimental Study for High Frequency Vibration Charac-Teristics of Duffing's Vibration Model Using Magnet-Spring," Proc. Dynamics and Design Conf., pp. 93-98, 2002.

[3] H. Sakuyama, S. Kojima, E. Fujita, S. Kaneko, M. Enokizono and K. Nishiyama, "Study of Suspension Seat us-ing Magnetic Spring and Magnetic Damper," Proc. JSME annual meeting, Vol. 7, pp. 35-36, 2006.

[4] M. Enokizono, T. Todaka, S. Nagata and E. Ishibashi, "Dynamic Finite Element Analysis of a Magnetic Damper System," IEEE Trans. Magnetics, Vol. 35, No. 5, pp. 3733-3735, 1999.

[5] K. Eguchi, S. Zeze, T. Todaka and M. Enokizono "Magnetic Filed Analysis of Magnetic Flux Concentration Type Hybrid Surface Magnet Motor," IEEJ Trans. FM, Vol. 130, No. 7, pp. 698-703, 2010. 